\title{
Association of Thyroid Status with Health-Related Quality of Life in Korean Older Adults
}

\author{
Minjung Han', Seulggie Choi ${ }^{2}$, Sarang Kim', Ahryoung Ko', Joung Sik Son', Sang Min Park'1,*** \\ 'Department of Family Medicine, Seoul National University Hospital, Seoul National University College of Medicine, Seoul, Korea \\ ${ }^{2}$ Department of Biomedical Sciences, Seoul National University College of Medicine, Seoul, Korea
}

Background: While hypothyroidism is associated with negative health effects in the general population, older adults with hypothyroidism have better physical function and comparable rates of depression and cognitive impairment relative to their euthyroid counterparts. The aim of this study was to investigate the association between thyroid status and health-related quality of life in Korean older adults.

Methods: In this population-based cross-sectional study, 1,060 adults aged over 60 years were classified by thyroid status into four groups based on their thyroid stimulating hormone (TSH) and free T4 values: overt hypothyroid, subclinical hypothyroid, euthyroid, and subclinical hyperthyroid. The main outcome measure was self-reported health-related quality of life based on the three-level version of the EuroQol-5 dimension (EQ-5D), with utility values of -0.171 and 1.000 corresponding to the worst and best health statuses, respectively. The adjusted means of the EQ-5D three-level version utility values according to thyroid status were determined using a linear regression analysis.

Results: In the adjusted analysis, the overt hypothyroid group showed significantly higher EQ-5D three-level version utility values than did the euthyroid group $(0.998$ vs. $0.908, \mathrm{P}=0.000)$. In the subgroup analyses by sex, the overt hypothyroid group also showed significantly higher EQ-5D three-level version utility values for both men and women than did the euthyroid group ( 0.998 vs. $0.940, \mathrm{P}=0.008 ; 0.983$ vs. $0.882, \mathrm{P}=0.001$ ).

Conclusion: Asymptomatic Korean older adults aged over 60 years with TSH and free T4 values corresponding to overt hypothyroidism have better health-related quality of life than their euthyroid counterparts.

Keywords: Hypothyroidism; Health-Related Quality of Life; Elderly

Received: May 31, 2019, Revised: July 15, 2019, Accepted: August 13, 2019

*Corresponding Author: Sang Min Park https://orcid.org/0000-0002-7498-4829

Tel: +82-2-2072-3331, Fax: +82-2-766-3276,E-mail: smpark@snuh.org 


\section{INTRODUCTION}

Hypothyroidism is a significant health problem that increases in frequency in the elderly. It affects approximately $4.6 \%$ of the US population (overt hypothyroidism, $0.3 \%$; subclinical hypothyroidism, $4.3 \%$ ) and $3.8 \%$ of the Korean population (overt hypothyroidism, $0.7 \%$; subclinical hypothyroidism, 3.1\%). ${ }^{1,2)}$ The frequency of subclinical hypothyroidism increases in older adults, affecting approximately $6 \%$ of individuals aged $70-79$ years and $10 \%$ of individuals aged 80 years or older. ${ }^{3)}$ The clinical manifestations of hypothyroidism include dry skin, cold sensitivity, fatigue, muscle cramps, voice changes, and constipation. ${ }^{4)}$ Hypothyroidism in the elderly also causes atypical symptoms, which include gustatory changes, hearing loss, and ataxia.

While the increased prevalence of hypothyroidism in the elderly is accompanied by higher rates of physical and cognitive impairments, depression, and cardiovascular diseases, hypothyroidism may have a protective health effect in older adults. ${ }^{3,5-9)}$ While reports differ, some studies have shown that older adults with thyroid stimulating hormone (TSH) levels that correspond to mild to overt hypothyroidism may have better mobility and slower decline in measures of physical function than their euthyroid counterparts. ${ }^{3,6)}$ Further, studies have shown that subclinical hypothyroidism in the elderly is not associated with depression or cognitive impairment. ${ }^{6,10,11)}$

To date, only a few studies have investigated the association between various degrees of hypothyroidism and measures of health-related quality of life in geriatric populations. The Korea National Health and Nutrition Examination Survey (KNHANES) represents a large and reliable dataset based on a nation-wide survey. Given the lack of studies that investigate the health effects of different thyroid statuses in geriatric populations and the importance of verifying their association, we assessed the cross-sectional relationship between thyroid status and EuroQol-5 dimension three-level version (EQ-5D-3L) utility values using data from the sixth KNHANES conducted from 2013 to 2015.

\section{METHODS}

\section{Study Population and Data Collection}

This study was conducted using data from the sixth KNHANES, a national cross-sectional survey conducted by the Korea Centers for Disease Control and Prevention for Health Statistics. The KNHANES is an independent dataset from the general South Korean population, similar to the National Health and Nutrition Examination Survey conducted by the US Centers for Disease Control and Prevention. ${ }^{12)}$ The selection process of household units and participants for the KNHANES is based on a stratified, multi-stage, clustered probability sampling design to represent the noninstitutionalized Korean population. The health interview includes the use of an established questionnaire to determine the demographical information of the subjects and basic laboratory data. In the sixth KNHANES (2013-2015), laboratory measurements of serum TSH, free T4 (fT4), and thyroid peroxidase antibody (TPO Ab) were obtained using subsampling stratified by sex and age. Approximately one-third of subjects aged over 10 years among a total of 2,400 individuals annually were selected by subsampling considering sociodemographic factors to undergo thyroid function tests.

Among the 1,195 subjects aged over 60 years who participated in the sixth KNHANES and underwent thyroid function tests, the following were excluded: those with missing EQ-5D-3L utility values $(n=74)$; those with self-reported history of thyroid diseases, including thyrotoxicosis, overt hypothyroidism, benign thyroid nodules, and Hashimoto's thyroiditis, and those who were on treatment for thyroid diseases, including radioactive iodine treatment, antithyroid drugs (propylthiouracil, methimazole, and carbimazole), and thyroid hormone (levothyroxine [LT4]) (n=38); those with a history of thyroid cancer $(\mathrm{n}=14)$; those with missing covariates $(\mathrm{n}=8)$; and those with overt hyperthyroidism (TSH level of $<0.10 \mathrm{mIU} / \mathrm{L}$ with elevated fT4 level) owing to the small patient number $(n=1)$. There were no pregnant individuals. The final study population included 1,060 individuals.

\section{Assessment of Thyroid Status}

The serum TSH, fT4, and TPO Ab levels were measured using electrochemiluminescence immunoassay (Roche Diagnostics, Mannheim, Germany) according to the criteria of the College of American Pathologists. In the main analysis, the Korean reference interval for the serum TSH level (0.62-6.68 mIU/L) was used, which is higher than that of Western countries. ${ }^{2}$ This reference range reflects the 2.5th and 97.5th percentile of the serum TSH levels of the reference population, defined as the population with no history of thyroid disease or thyroid cancer and no history of taking medications that could influence thyroid function, with no family history of thyroid disease, with negative TPO Ab findings, and with serum fT4 levels within the reference range (0.89$1.76 \mathrm{ng} / \mathrm{mL}$ ), as previously reported; this population did not include pregnant women. ${ }^{2)}$ In the supplemental analysis, thyroid status was classified using the manual TSH reference range from Roche Diagnostics (0.35-5.50 mIU/L). The manual reference range for the serum fT4 level (0.89-1.76 ng/mL) was used in both main and supplemental analyses. The reference range for the TPO Ab level was $<34 \mathrm{IU} / \mathrm{mL}$.

\section{Classification by Thyroid Status}

As described in a previous study, the subjects were classified into the following groups according to their thyroid function test results. ${ }^{13)}$ Using the population-based TSH reference range (0.62-6.68 mIU/L), thyroid status was classified as follows: overt hypothyroidism was defined as a TSH level of $20 \mathrm{mIU} / \mathrm{L}$ or more or a TSH level of more than 6.68 $\mathrm{mIU} / \mathrm{L}$ with an fT4 level below normal $(<0.89 \mathrm{ng} / \mathrm{mL})(\mathrm{n}=10)$; subclinical hypothyroidism was defined as a TSH level of more than $6.68 \mathrm{mIU} /$ $\mathrm{L}$ and less than $20 \mathrm{mIU} / \mathrm{L}$ with a normal fT4 level (0.89-1.76 ng/mL) $(\mathrm{n}=31)$; euthyroid was defined as a normal TSH level (0.62-6.68 mIU/ L) (n=987); subclinical hyperthyroidism was defined as a TSH level of 0.10-0.6199 mIU/L or less than $0.10 \mathrm{mIU} / \mathrm{L}$ with a normal fT4 level $(\mathrm{n}=32)$; and overt hyperthyroidism was defined as a TSH level of less than $0.10 \mathrm{mIU} / \mathrm{L}$ with an elevated fT4 level $(\mathrm{n}=1)$. The subject with overt hyperthyroidism $(n=1)$ was excluded owing to the small patient 
number. In the supplemental analysis, thyroid status was classified in a similar manner using the manual TSH reference range from Roche Diagnostics (0.35-5.50 mIU/L).

\section{Assessment of the EuroQol-5 Dimension Three-Level Version Utility Values}

Health-related quality of life was assessed using the EQ-5D-3L, a generic preference-based measure consisting of five questions concerning the current health status of patients. ${ }^{14)}$ The questions target five areas: mobility, self-care, usual activities, pain/discomfort, and anxiety/ depression. Each question has three possible responses, i.e., no problem, some problems, or severe problems. Population-specific value sets used for deriving utility values from responses to questions enable effective comparison across different populations. The EQ-5D-3L has been widely used as a standard measure of health-related quality of life. The Korean EQ-5D-3L was developed by the Korea Centers for Disease Control and Prevention according to procedures outlined by the EuroQol group. The utility values for the Korean EQ-5D-3L range continuously from -0.171 (worst health status) to 1.000 (best health status).

\section{Key Variables and Statistical Analysis}

The considered covariates included age (years; continuous), sex (male or female; categorical), body mass index ( $\mathrm{kg} / \mathrm{m}^{2}$; continuous), household income (lower half or upper half; categorical), education ( $\leq 6$ years or $>6$ years; categorical), employment status (unemployed or employed; categorical), smoking status (nonsmoker or current and past smoker; categorical), drinking status (nondrinker or drinker; categorical), physical activity (meets or does not meet requirements; categorical), diabetes (with or without; categorical), hypertension (with or without; categorical), dyslipidemia (with or without; categorical), serum hemoglobin level (mg/dL; continuous), and serum TPO Ab level (normal or high; categorical). Physical activity was calculated as metabolic equivalent task (MET) minutes per week using the International Physical Activity Questionnaire in 2013 and Global Physical Activity Questionnaire in 2014 and 2015. ${ }^{15,16)}$ It was classified as not meeting the requirements ( $<600 \mathrm{MET}-\mathrm{min} / \mathrm{wk}$ ) or meeting the requirements ( $\geq 600$ MET-min/wk). Further, the TPO Ab levels were classified into normal $(\leq 34 \mathrm{IU} / \mathrm{mL})$ or high $(>34 \mathrm{IU} / \mathrm{mL})$.

The chi-square test was used for categorical variables and one-way analysis of variance for continuous variables to test for significant dif-

Table 1. Descriptive characteristics of the study population (population-based reference range)

\begin{tabular}{|c|c|c|c|c|c|c|}
\hline Characteristic & Category & Overt hypothyroid & $\begin{array}{l}\text { Subclinical } \\
\text { hypothyroid }\end{array}$ & Euthyroid & $\begin{array}{l}\text { Subclinical } \\
\text { hyperthyroid }\end{array}$ & P-value \\
\hline No. of participants & & 10 & 31 & 987 & 32 & \\
\hline Age $(y)$ & & $65 \pm 3.9$ & $66.1 \pm 4.6$ & $65.4 \pm 3.8$ & $65.5 \pm 4.9$ & 0.778 \\
\hline \multirow[t]{2}{*}{ Sex } & Male & $4(40.0)$ & $11(35.5)$ & $510(51.7)$ & $17(53.1)$ & 0.296 \\
\hline & Female & $6(60.0)$ & $20(64.5)$ & $477(48.3)$ & $15(46.9)$ & \\
\hline \multirow[t]{2}{*}{ Body mass index } & Nonobese & $3(30.0)$ & $22(71.0)$ & $618(62.6)$ & $17(53.1)$ & 0.085 \\
\hline & Obese & $7(70.0)$ & $9(29.0)$ & $369(37.4)$ & $15(46.9)$ & \\
\hline \multirow[t]{2}{*}{ Household income } & Lower half & $6(60.0)$ & $15(48.4)$ & $614(62.2)$ & $21(65.6)$ & 0.450 \\
\hline & Upper half & $4(40.0)$ & $16(51.6)$ & $373(37.8)$ & $11(34.4)$ & \\
\hline \multirow[t]{2}{*}{ Education } & $\leq 6 \mathrm{y}$ & $5(50.0)$ & $14(45.2)$ & $475(48.1)$ & $17(53.1)$ & 0.932 \\
\hline & $>6 y$ & $5(50.0)$ & $17(54.8)$ & $512(51.9)$ & $15(46.9)$ & \\
\hline \multirow[t]{2}{*}{ Employment status } & Unemployed & $8(80.0)$ & $18(58.1)$ & $511(51.8)$ & $16(50.0)$ & 0.301 \\
\hline & Employed & $2(20.0)$ & $13(41.9)$ & $476(48.2)$ & $16(50.0)$ & \\
\hline \multirow[t]{2}{*}{ Smoking status } & Nonsmoker & $7(70.0)$ & $24(77.4)$ & $520(52.7)$ & $16(50.0)$ & 0.034 \\
\hline & Current and past smoker & $3(30.0)$ & 7 (22.6) & $467(47.3)$ & $16(50.0)$ & \\
\hline \multirow[t]{2}{*}{ Drinking status } & Nondrinker & $4(40.0)$ & $17(54.8)$ & $364(36.9)$ & $12(37.5)$ & 0.244 \\
\hline & Drinker & $6(60.0)$ & $14(45.2)$ & $623(63.1)$ & $20(62.5)$ & \\
\hline \multirow[t]{2}{*}{ Physical activity* } & Does not meet requirements & $2(20.0)$ & 19 (61.3) & $481(48.7)$ & $21(65.6)$ & 0.033 \\
\hline & Meets requirements & $8(80.0)$ & $12(38.7)$ & $506(51.3)$ & $11(34.4)$ & \\
\hline \multirow[t]{2}{*}{ Hypertension } & No & $8(80.0)$ & $18(58.1)$ & $562(56.9)$ & $17(53.1)$ & 0.499 \\
\hline & Yes & $2(20.0)$ & $13(41.9)$ & $425(43.1)$ & $15(46.9)$ & \\
\hline \multirow[t]{2}{*}{ Dyslipidemia } & No & $8(80.0)$ & $21(67.7)$ & $713(72.2)$ & $26(81.3)$ & 0.595 \\
\hline & Yes & $2(20.0)$ & $10(32.3)$ & $274(27.8)$ & $6(18.7)$ & \\
\hline \multirow[t]{2}{*}{ Diabetes } & No & $8(80.0)$ & $27(87.1)$ & $813(82.4)$ & $27(84.4)$ & 0.900 \\
\hline & Yes & $2(20.0)$ & $4(12.9)$ & $174(17.6)$ & $5(15.6)$ & \\
\hline Hemoglobin level & & $13.2 \pm 0.8$ & $13.4 \pm 1.1$ & $14.0 \pm 1.3$ & $13.9 \pm 1.3$ & 0.011 \\
\hline \multirow[t]{2}{*}{ Thyroid peroxidase antibody level ${ }^{\dagger}$} & Normal & $8(80.0)$ & $27(87.1)$ & $906(91.8)$ & $30(93.8)$ & 0.422 \\
\hline & High & $2(20.0)$ & $4(12.9)$ & $81(8.2)$ & $2(6.2)$ & \\
\hline EuroQol three-level version & & $0.9497 \pm 0.1029$ & $0.8985 \pm 0.1004$ & $0.9063 \pm 0.1397$ & $0.8844 \pm 0.1316$ & 0.599 \\
\hline
\end{tabular}

Values are presented as number, mean \pm standard deviation, or number (\%).

MET, metabolic equivalent task.

*Does not meet requirements: <600 MET-min/wk; meets requirements: $\geq 600 \mathrm{MET}-\mathrm{min} / \mathrm{wk}$. ${ }^{\dagger}$ Normal: $\leq 34 \mathrm{IU} / \mathrm{mL}$; high: > $34 \mathrm{IU} / \mathrm{mL}$. 


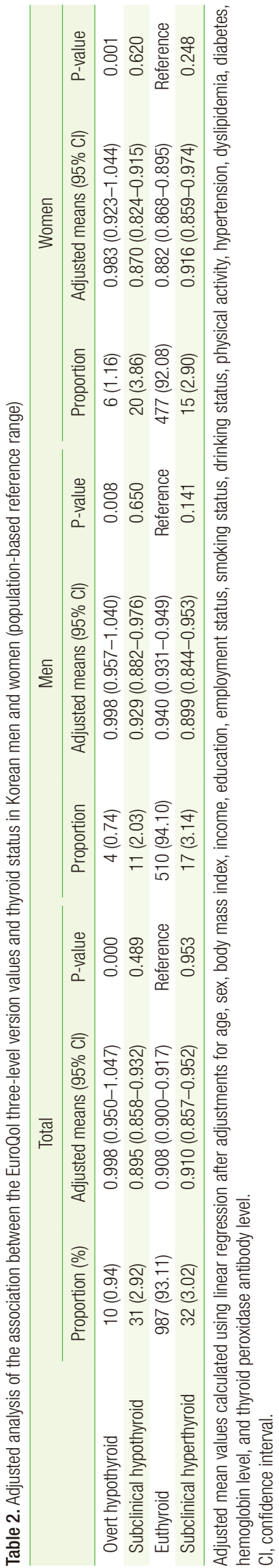

ferences in the means across the different thyroid status groups. Subsequently, an adjusted linear regression analysis was performed to test for significant differences in the EQ-5D-3L values across the sample groups (Table 2). Ninety-five percent confidence intervals were applied, and P-values of $<0.05$ were considered statistically significant for all results. All statistical analysis was performed using STATA ver. 14.0 (STATA Corp., College Station, TX, USA).

\section{Ethics Statement}

Prior to the survey, all participants of the sixth KNHANES provided informed consent. As the national survey data used for this study were publicly available from the Korea Centers for Disease Control and Prevention, no ethical approval from our Institutional Review Board was necessary.

\section{RESULTS}

\section{Characteristics of the Study Population}

The study subjects were a disease-free population $(n=1,060)$ whose baseline characteristics are shown in Table 1. Their mean age was 65.4 years, and the proportion of men was $51.1 \%$. There were 10 subjects in the overt hypothyroid group, 31 subjects in the subclinical hypothyroid group, 987 individuals in the euthyroid group, and 32 subjects in the subclinical hyperthyroid group (Table 1). Significant differences in the means were observed across the groups only for smoking status, physical activity, and hemoglobin levels $(\mathrm{P}=0.034,0.033,0.011$, respectively). Across the sample groups, the overt hypothyroid group demonstrated the highest unadjusted mean EQ-5D-3L utility value, although the association was not significant $(0.9497, \mathrm{P}=0.599)$.

\section{Adjusted Mean EuroQol-5 Dimension Three-Level Version Utility Values according to Thyroid Status}

In the adjusted analysis, the overt hypothyroidism group had significantly higher mean least-square EQ-5D-3L utility values than the euthyroid group in the total population as well as in the male and female subpopulations ( 0.998 versus $0.908,0.998$ versus $0.940,0.983$ versus 0.882; $\mathrm{P}=0.000,0.008,0.001$, respectively) (Table 2). At baseline of euthyroid, neither the subclinical hypothyroid group nor the subclinical hyperthyroid group showed a significant association (Table 2, Figure $1)$.

\section{Categorization based on Population-Specific (0.62-6.68 mIU/L) versus Manual-Based Thyroid Stimulating Hormone Reference Ranges (0.35-5.50 mIU/L)}

When thyroid status was classified using the manual-based TSH reference range (0.35-5.50 mIU/L) instead of the population-based TSH reference range $(0.62-6.68 \mathrm{mIU} / \mathrm{L})$, the distribution of individuals in each group shifted slightly (Supplementary Table 1). There were 11 subjects in the overt hypothyroid group, 61 subjects in the subclinical hypothyroid group, 977 subjects in the euthyroid group, and 11 subjects in the subclinical hyperthyroid group (Supplementary Table 1). 
A

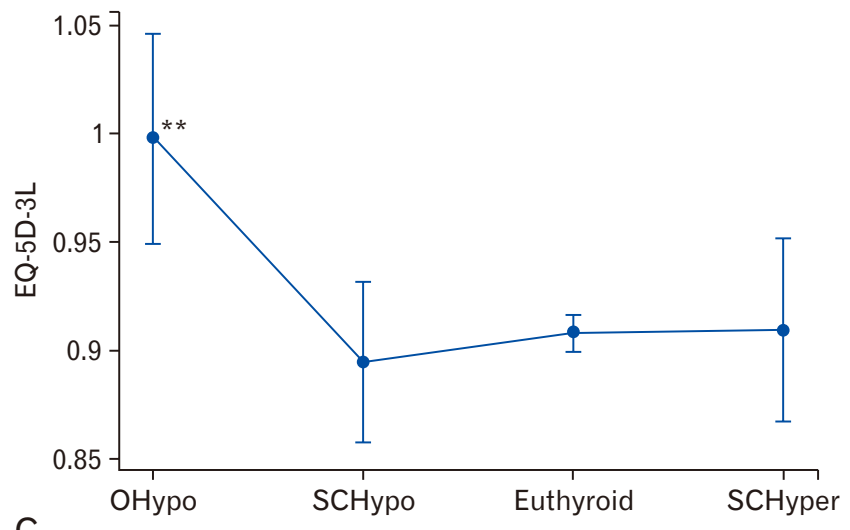

C

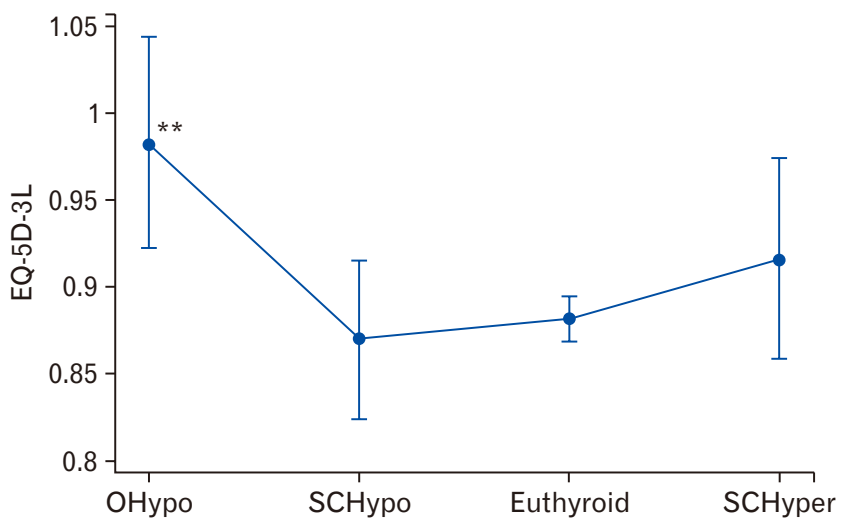

B

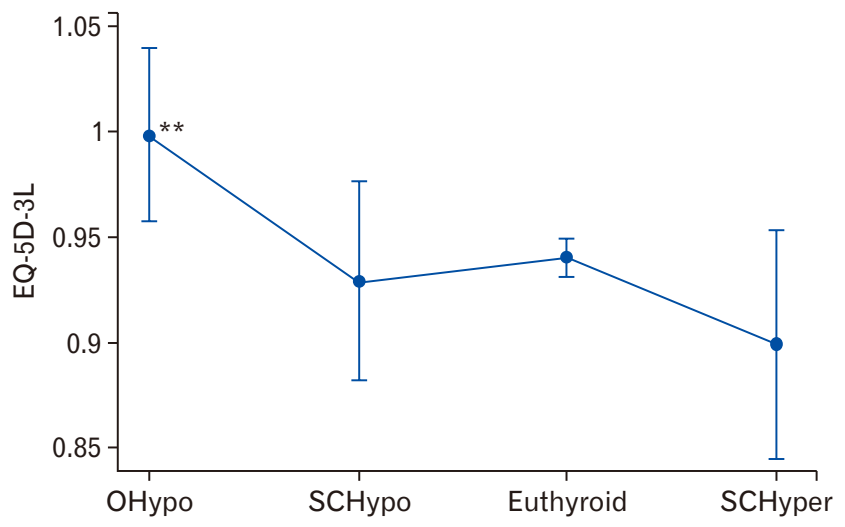

Figure 1. Association between health-related quality of life and thyroid status at baseline of euthyroid (population-based TSH reference range). Adjusted mean EQ5D-3L utility scores calculated using the linear regression model analysis in the total population (A), men (B), and women (C). EQ-5D-3L: adjusted mean utility scores based on the Korean EQ-5D-3L index (normal range, -0.171 to 1.000 ); populationbased TSH reference range: 0.62 to $6.68 \mathrm{mlU} / \mathrm{L}$. TSH, thyroid stimulating hormone; EQ-5D-3L, EuroQol three-level version; OHypo, overt hypothyroid; SCHypo, subclinical hypothyroid; SCHyper, subclinical hyperthyroid. ${ }^{*} P<0.05 .{ }^{* *} P<0.01$.

Significant differences were observed across the groups only for smoking, drinking and hemoglobin levels (Supplementary Table 1). The unadjusted mean EQ-5D-3L utility values were the highest in the overt hypothyroid group, although the association was not significant $(\mathrm{P}=0.714)$ (Supplementary Table 1). In the adjusted analysis, the mean least-square EQ-5D-3L utility values increased in the overt hypothyroid group relative to the euthyroid group, although the association was not significant ( 0.968 versus $0.908, \mathrm{P}=0.106$ ) (Supplementary Table 2, Supplementary Figure 1).

\section{DISCUSSION}

In this nationwide, population-based, cross-sectional study using the sixth KNHANES data, we showed that a significant association might exist between overt hypothyroidism and higher EQ-5D-3L utility values in Korean subjects aged over 60 years (Table 2). This result suggests that asymptomatic older adults with TSH and fT4 levels that correspond to overt hypothyroidism might have better health-related quality of life than their euthyroid counterparts.

Several previous studies have reported on the relationship between hypothyroidism and measures of health-related quality of life in older adults, including mobility, self-care, usual activity, pain/disability, and anxiety/depression. For instance, a prospective population-based study conducted in participants followed up from age 85 to 89 years showed that increasing levels of TSH at baseline were associated with significant decelerated increases in disability in instrumental activities of daily living. ${ }^{6)}$ Another prospective population-based study of healthy asymptomatic older adults aged 70 to 79 years showed that their mild subclinical hypothyroid group demonstrated better mobility and had a higher percentage of those with good cardiorespiratory fitness and who reported walking in ease than did their euthyroid group. ${ }^{3)}$ Finally, a study using data from the Korean Longitudinal Study on Health and Aging on Korean subjects aged over 65 years found that subclinical hypothyroidism was not associated with cognitive impairment, depression, or poor quality of life in the elderly. ${ }^{11)}$ Some of these studies differ from our study in that the study population consisted of patients with subclinical hypothyroidism, rather than those with overt hypothyroidism. Moreover, these studies differed from our study in terms of the age of the study population.

Several mechanisms can possibly explain our finding that older adults aged over 60 years with overt hypothyroidism have better health-related quality of life than their euthyroid counterparts. Thyroid hormone plays a critical role in energy metabolism. Overt hypothyroidism may slow the metabolic rate, which may result in increased preservation of physical and cognitive functions. ${ }^{6}$ This protective effect of overt hypothyroidism has been reported in the oldest old aged at least 70 years. ${ }^{5)}$ In young and middle-aged humans, the negative effects of hypothyroidism, such as dyslipidemia and adverse cardiovas- 
cular outcomes, are more dominant. ${ }^{5)}$

Clinical practices concerning the treatment of hypothyroidism in the elderly are mostly similar in the United States and in Korea. Screening for hypothyroidism is performed by measuring the serum TSH level. ${ }^{17,18)}$ Treatment with LT4 is recommended for symptomatic older adults with persistently elevated TSH levels and decreased fT4 levels. ${ }^{18,19)}$ The dose of LT4 depends on such factors as the presence and severity of thyroid-related symptoms and coexisting diseases, e.g., coronary artery disease and heart failure. ${ }^{18,19)}$ Older adults without risks of heart diseases may be initiated on a full dose of LT4 (1.6 mcg/ $\mathrm{kg} / \mathrm{d}$ ), while those with risks are started on a lower dose $(25-50 \mathrm{mcg} / \mathrm{d})$ that is increased gradually. ${ }^{18,19)}$ For subclinical hypothyroidism, treatment remains controversial, as no benefit was observed with treating patients aged over 70 years. ${ }^{17,18,20-23)}$

While the target TSH reference range is $0.5-4.5 \mathrm{mIU} / \mathrm{L}$ for the general population, it is recommended that the target serum TSH level be set to approximately $4-6 \mathrm{mIU} / \mathrm{L}$ in individuals aged over 70 years, as the TSH levels tend to increase in older adults even without thyroid disease. ${ }^{17,18)}$ It is important to determine the optimal TSH target levels, as the elderly are especially susceptible to the adverse effects of excess LT4, such as atrial fibrillation and osteoporotic fractures. ${ }^{18)}$ In both the United States and Korea, there is a lack of randomized controlled studies of LT4 treatment in elderly patients with hypothyroidism comparing different TSH target values. ${ }^{18,19)}$ By showing that healthy asymptomatic Korean older adults without evident thyroid disease experience better quality of life at elevated TSH levels, our study highlights the importance of determining population-specific TSH target values for LT4 treatment in older adults.

Our study is the first to utilize data from the sixth KNHANES to investigate the association between thyroid status and health-related quality of life. One strength of our study is that as our results were derived from a large and reliable national dataset, they are more likely to be representative of the findings of the general population. Furthermore, we used both population-specific and manual-based reference ranges for TSH in our analyses. It has been reported that TSH levels vary across different geographic and ethnic groups. ${ }^{2)}$ According to a previous KNHANES study, the Korean reference population demonstrates higher TSH levels than does the US population, which is most likely attributed to the fact that Korea is an iodine-sufficient region. ${ }^{1,2)}$ Our study demonstrates that the association between overt hypothyroidism and EQ-5D-3L values is maintained even when the manualbased TSH reference range is used, although the statistical significance is lost (Supplementary Table 2).

One limitation of our study is that as it is based on cross-sectional data, we only observed TSH levels measured on a single occasion. For the same reason, we could not follow up on participants to investigate the effect of thyroid status on long-term outcomes or determine cause and effect. Further, owing to the small sample size of the overt hypothyroid group, which accounted for less than $1 \%$ of the total study population, our results, while statistically significant, are not sufficient to make any definitive statements and need to be substantiated by fur- ther research. As we performed our analyses based on data from the Korean population, it is unknown whether our conclusions will apply to other racial populations. Lastly, we were not able to measure the components of EQ-5D-3L, such as physical performance, directly.

In conclusion, our study suggests that overt hypothyroidism in older adults might be significantly associated with improved health-related quality of life. Moreover, several previous studies have reported that asymptomatic older adults with thyroid function test findings corresponding to overt or subclinical hypothyroidism tend to have better physical function and comparable rates of depression and cognitive impairment relative to their euthyroid counterparts. Thus, further studies should be conducted to investigate the possible protective health effect of hypothyroidism in older adults.

\section{CONFLICT OF INTEREST}

No potential conflict of interest relevant to this article was reported. Seoul National University Hospital had no role in the design and conduct of the study; collection, management, analysis, and interpretation of the data; preparation, review, or approval of the manuscript; and decision to submit for publication.

\section{ORCID}

Minjung Han: https://orcid.org/0000-0002-9311-5370

Seulggie Choi: https://orcid.org/0000-0002-0581-165X

Sarang Kim: https://orcid.org/0000-0001-5120-6357

Ahryoung Ko: https://orcid.org/0000-0001-8381-5595

Joung Sik Son: https://orcid.org/0000-0003-2460-3378

Sang Min Park: https://orcid.org/0000-0002-7498-4829

\section{REFERENCES}

1. Hollowell JG, Staehling NW, Flanders WD, Hannon WH, Gunter EW, Spencer CA, et al. Serum TSH, T(4), and thyroid antibodies in the United States population (1988 to 1994): National Health and Nutrition Examination Survey (NHANES III). J Clin Endocrinol Metab 2002;87:489-99.

2. Kim WG, Kim WB, Woo G, Kim H, Cho Y, Kim TY, et al. Thyroid stimulating hormone reference range and prevalence of thyroid dysfunction in the Korean population: Korea National Health and Nutrition Examination Survey 2013 to 2015. Endocrinol Metab (Seoul) 2017;32:10614.

3. Simonsick EM, Newman AB, Ferrucci L, Satterfield S, Harris TB, Rodondi N, et al. Subclinical hypothyroidism and functional mobility in older adults. Arch Intern Med 2009;169:2011-7.

4. Garber JR, Cobin RH, Gharib H, Hennessey JV, Klein I, Mechanick JI, et al. Clinical practice guidelines for hypothyroidism in adults: cosponsored by the American Association of Clinical Endocrinologists and the American Thyroid Association. Endocr Pract 2012;18:9881028.

5. Cooper DS. Thyroid disease in the oldest old: the exception to the rule. 
JAMA 2004;292:2651-4.

6. Gussekloo J, van Exel E, de Craen AJ, Meinders AE, Frolich M, Westendorp RG. Thyroid status, disability and cognitive function, and survival in old age. JAMA 2004;292:2591-9.

7. Davis JD, Stern RA, Flashman LA. Cognitive and neuropsychiatric aspects of subclinical hypothyroidism: significance in the elderly. Curr Psychiatry Rep 2003;5:384-90.

8. Dugbartey AT. Neurocognitive aspects of hypothyroidism. Arch Intern Med 1998;158:1413-8.

9. Gulseren S, Gulseren L, Hekimsoy Z, Cetinay P, Ozen C, Tokatlioglu B. Depression, anxiety, health-related quality of life, and disability in patients with overt and subclinical thyroid dysfunction. Arch Med Res 2006;37:133-9.

10. Hong JW, Noh JH, Kim DJ. Association between subclinical thyroid dysfunction and depressive symptoms in the Korean adult population: the 2014 Korea National Health and Nutrition Examination Survey. PLoS One 2018;13:e0202258.

11. Park YJ, Lee EJ, Lee YJ, Choi SH, Park JH, Lee SB, et al. Subclinical hypothyroidism (SCH) is not associated with metabolic derangement, cognitive impairment, depression or poor quality of life (QoL) in elderly subjects. Arch Gerontol Geriatr 2010;50:e68-73.

12. Kweon S, Kim Y, Jang MJ, Kim Y, Kim K, Choi S, et al. Data resource profile: the Korea National Health and Nutrition Examination Survey (KNHANES). Int J Epidemiol 2014;43:69-77.

13. Cappola AR, Fried LP, Arnold AM, Danese MD, Kuller LH, Burke GL, et al. Thyroid status, cardiovascular risk, and mortality in older adults. JAMA 2006;295:1033-41.

14. Mozzi A, Meregaglia M, Lazzaro C, Tornatore V, Belfiglio M, Fattore G. A comparison of EuroQol 5-dimension health-related utilities using
Italian, UK, and US preference weights in a patient sample. Clinicoecon Outcomes Res 2016;8:267-74.

15. Craig CL, Marshall AL, Sjostrom M, Bauman AE, Booth ML, Ainsworth $\mathrm{BE}$, et al. International physical activity questionnaire: 12-country reliability and validity. Med Sci Sports Exerc 2003;35:1381-95.

16. World Health Organization. Global physical activity questionnaire (GPAQ) analysis guide. Geneva: World Health Organization; 2012.

17. Kim JH. Thyroid dysfunction in the elderly. J Korean Thyroid Assoc 2012;5:94-8.

18. Jonklaas J, Bianco AC, Bauer AJ, Burman KD, Cappola AR, Celi FS, et al. Guidelines for the treatment of hypothyroidism: prepared by the American Thyroid Association task force on thyroid hormone replacement. Thyroid 2014;24:1670-751.

19. Park KH, Lee EJ. Recent review on medical treatment of thyroid disease. J Korean Med Assoc 2012;55:1207-14

20. Parle J, Roberts L, Wilson S, Pattison H, Roalfe A, Haque MS, et al. A randomized controlled trial of the effect of thyroxine replacement on cognitive function in community-living elderly subjects with subclinical hypothyroidism: the Birmingham Elderly Thyroid Study. J Clin Endocrinol Metab 2010;95:3623-32.

21. Roberts LM, Pattison H, Roalfe A, Franklyn J, Wilson S, Hobbs FD, et al. Is subclinical thyroid dysfunction in the elderly associated with depression or cognitive dysfunction? Ann Intern Med 2006;145:573-81.

22. Takashima N, Niwa Y, Mannami T, Tomoike H, Iwai N. Characterization of subclinical thyroid dysfunction from cardiovascular and metabolic viewpoints: the Suita study. Circ J 2007;71:191-5.

23. Biondi B, Cooper DS. The clinical significance of subclinical thyroid dysfunction. Endocr Rev 2008;29:76-131. 